\title{
Prediction of Hybrid Performance in Crop Plants: Molecular and Recent Approaches
}

\author{
Sandeep Kumar Soni ${ }^{1 *}$, Shailesh Tiwari², John T. Newmah ${ }^{3}$, \\ Innocent Dossou-Aminon ${ }^{4}$ and R.M. Sundaram ${ }^{5}$
}

\author{
${ }^{1}$ Department of Genetics and Plant Breeding, C. S. Azad University of Agriculture and \\ Technology, Kanpur, U.P., India \\ ${ }^{2}$ Division of Genomic Resources, National Bureau of Plant Genetic Resources, Pusa Campus, \\ New Delhi, India \\ ${ }^{3}$ Faculty of Cereal Crop Breeding, Central Agricultural Research Institute, Bong, Liberia \\ ${ }^{4}$ Faculty of Science and Technology of Dassa Polytechnic University of Abomey, \\ Dassa-Zoumè, Benin \\ ${ }^{5}$ Indian Institute of Rice Research, Rajendranagar, Hyderabad, Telangana, India
}

*Corresponding author

\section{A B S T R A C T}

\begin{tabular}{|l|}
\hline Ke y w o r d s \\
Heterotic QTLs, \\
Wider \\
compatibility, \\
Neutral alleles, \\
Transcriptomics and \\
high definition \\
genotyping
\end{tabular}

Hybrid breeding technology is one of the most feasible options to meet the future food challenges and sustainable agriculture. Large number of cross combinations are attempted and evaluated in hybrid breeding programs, but a few are selected. This is labour intensive, time consuming and costly; despite of it selection efficiency is too low. Therefore, it is important to explore alternate approaches to enhance the selection efficiency to predict hybrid performance. Pedigree data of the parental lines and molecular marker have been effectively employed to predict field performance of hybrids. Marker technologies, wider gene compatibility and QTL approaches on the basis of parental genomic variation are predominantly used for predicting hybrid performance. Informative markers having high PIC would be considered as key markers for heterotic performance prediction. Prediction ability depends on association between QTL and selected molecular markers with major effect on the desired trait, size of population and the recombination frequencies. Transcriptomic and metabolomics data are becoming useful and may provide means for heterosis prediction. Joint analysis of the genomic, transcriptomic and metabolomics data for molecular-based prediction of heterosis needs to be studied for its potential to improve the efficiency of applied hybrid breeding programs. Inter-sub-specific cross combinations depends on genetic remedy to the persisting hybrid semi-sterility problem and entire diversity, and present of neutral wide compatible genes.

\section{Introduction}

In the $21^{\text {st }}$ century, great progress has been made for food security by developing superior hybrid varieties, biomass security in dual purpose crops, disease resistance and crop quality. High fertilizer responsiveness, pesticide availability, better varieties from 
existing ones and adapted crop husbandry systems have made these steps forward possible. On the other hand intensive agriculture has severe drawbacks such as loss of biodiversity, smaller crop rotations, higher degree of disease pressure, lower soil fertility and worsened soil structure. In the future, breeders will have to focus on revolutionizing these negative effects following systematic breeding programme while maintaining high productivity. In general, achieving the higher productivity heterosis breeding is one of the feasible techniques to meet the future food demands. To overcome the problem marker dependent technology is most feasible tool to meet the future challenges. Availability of diverse type of germplasm made breeder's task easy for development of superior hybrids by availing the crop reservoir. Molecular marker based heterosis prediction provides an alternative to expensive field testing for identification of superior hybrids and can greatly accelerate hybrid breeding programmes (Chen et al., 2013). In recent years marker technology significantly facilitated the development of successful crop hybrids by providing the means for selection of promising lines foreground, background selection system and quick development of haploids. We present, here a consigned outlook on techniques and the future approaches for HP, with a focus on the amalgamation of high-throughput genotyping and phenotyping platforms in hybrid breeding programs. Molecular marker techniques enabled to proliferate the hypothetic to explain the phenomenon involved in complementation of slightly deleterious recessive mutations (Charlesworth and Willis, 2009). Performance prediction of different crop parental lines is commonly organized based attempted crosses of genetically divergent heterotic groups, and commercial hybrid varieties are commonly associated with closed groups (Delucchi et al., 2012 and Kashiani et al., 2012).

\section{Factors influencing predicibility}

Heterosis is a well-known complicated biological phenomenon that is influenced by the mode of pollination, genomic diversity, genetic base, adaptability and several factors like inheritance of desired trait, method or experimental mating design, system of molecular marker, number of markers, informativeness of used markers, genomic coverage of the selected markers etc.

\section{Crop and plant material}

Self-pollinated crop species exhibit lower magnitude of buffering capacity as compared to cross pollinated ones due to the continuous selfing or submitting. Generally, heterosis level is known to be low in self-pollinated crop than cross pollinated crops. The magnitude of association is dependent of parental population size diversity and ploidy levels, also affects the association assay between genetic distances with heterosis value (Soni et al., 2017).

\section{Trait inheritance nature}

Mostly economic traits of crops plants are polygenic in nature, which are influenced by growing area and favourable environmental conditions. Heritability as well as heterosis expression level of the traits can be influenced by the genetic trait.

Some traits are governed by the one or two major genes such traits are less influenced by the environment. Association value between genetic diversity and heterosis, estimated would be better for the traits coupled with higher heredity than those of with lower. Similarly, lower levels of heterosis were observed for traits of interest in intra group than in inter groups of rice hybrids (Soni et $a l ., 2017)$. Association study in maize diallel cross concluded, that some QTLs located in 
the vicinity of loci having quantitative effect on grain yield (Vuylsteke, 2000).

\section{Selection of marker tool}

Numerous literatures are available for assessment of heterotic performance based on DNA markers in cultivated crops. Many coworkers supported, molecular markers could be used for heterosis prediction (Jaikishan et al., 2010 and Soni et al., 2017), while others disagreed (Chen et al., 2010 and $\mathrm{Wu}$ et al., 2013). However, technology proven a powerful tool for reliable heterosis prediction. Molecular markers varied in predictability and prediction is generally, better for the traits with high heritability than with poor ones. Inadequate genome coverage and different levels of dominance among hybrids may be one of the reasons for the low correlation between hybrid performance and marker diversity Choukan and Warburton (2006). Reis et al., (2005) suggested better prediction working on maize by integrating AFLP molecular marker and phenotypic data. Predictivenes of marker can be estimated based on PIC, which reflects the amount of polymorphism at given locus Micic et al., (2007). Jaikishan et al., (2010) utilized the markers with higher PIC value for EST-SSRs markers and reported markers for successful utilization for prediction of heterosis.

\section{Number of markers}

Heterosis prediction is more reliable with considering large number of molecular markers, uniformly distributed across the genome (Jaikishan et al., 2010 and Soni 2017). Shieh and Thseng (2002) worked with few markers and suggested low scope of predictive value because of inadequate genome coverage, selection of markers from non-coding region and different levels of dominance among hybrids may be one of the reasons for the low correlation between hybrid performance and marker diversity. The precision of heterotic performance depends on the adequate number of markers consideration tightly linked with coding region, wide distribution within the genome (Soni et al., 2017).

\section{Prediction method}

To predict the marker effectiveness, researchers need to develop large number of hybrids, estimate of combining ability and heterosis to identify the best hybrid. Breeders are always interested in choosing the superior lines among the potential parents which would result better heterotic combination. Trait based markers for heterosis prediction and parameters used to determine the genetic distances significantly affect the association level due to differential genetic basis of inheritance and measurement. Total markers based prediction "highly informative" was better for prediction based on effect increasing loci than more effective of total loci with individual positive loci.

\section{Approaches of heterosis prediction}

In present decades, major research threats has been noted for maximum utilization of mini core collections and assessing effect in the hybrid breeding programs for prediction of heterosis. Heterosis prediction helps to make early decision of superior parent selection for involving in hybrid breeding programme. It also accelerates in yield, agronomic and important economic traits prediction.

\section{Molecular marker based heterosis prediction}

\section{Genetic similarity/distance approach}

Parental genetic diversity has been realized, as natural reservoirs for the long-term breeding. Genetic distance between lines, 
obtained through molecular markers, has been considered best approach for prediction of hybrid performance. Usatov et al., (2014) suggested whole growth period, economic parameters of mid-parent advantage and the competitive advantage were significantly correlated with the genetic distances calculated with RAPD and SSR markers. Rajendran et al., (2014) noted genetic distances in rice were highly to moderately correlate with heterosis, while significant proportion of the heterosis was recorded with GD while other traits are more difficult to predict (Zanaty et al., 2013). Predicting heterosis with the estimates of genetic distance (GD) and genetic similarity (GS) between the parental lines based on randomly selected genomic DNA markers set were not successful for interspecific hybrids due to very poor linkage of heterotic QTLs. DNA markers have been utilized with the objective of predicting the performance of hybrids by genotyping of the progenies (Selvaraj et al., 2010). Associaation results are generally inconsistent regarding with the efficiency of hybrid since intermediate magnitude was also recorded hybrids and GD between their progenies.

\section{Marker polymorphism and heterosis association approach}

Moderate magnitude of association between heterosis and CMP value for marker traits was reported by Soni et al., (2017) in japonica and indica testers. Significant and positive correlation between experimental heterosis and SSR, EST-SSR was established and recorded more useful with informative ESTSSR (Jaikishan, 2010). Heterosis prediction estimated by employing GD SSRs and GD COPs association in $F_{1}$, produced by 20 parents revealed positive association with yield heterosis (Sud et al., 2010). Significant and positive correlation between the total number of bands and economic yield was also reported by Rabbani (2010). A low magnitude of correlations of MRD with hybrid yield was reported through bin wise chosen random markers in maize and for markers that were significantly associated with the trait also.

\section{GD and grain yield association approach}

Low correlations of GD with yield of hybrids, using random molecular markers were tested in 28 rice hybrids derived from 14 restorer and two CMS lines (Heffner, et al., 2001). Yu et al., 2005 used six CMS $\times$ five restorer lines in oilseed rape, among several markers (morphological, isozymes, soluble proteins, RAPD) and found increased the magnitude of correlations between GD and yield. A significant linear correlation was reported between the yield heterosis and marker-based genetic distance of parent, suggested that genetic distance could not accurately predict hybrid performance unless the DNA markers used in the analysis were linked to the stretch of genes affecting the trait (Xangsayasane et al., 2010 and Singh et al., 2011)

\section{Estimates of marker effect}

Molecular markers linked with hybrid yield and their allelic effects can be employed for prediction of heterosis. Association with large numbers of AFLP markers with heterosis for yield in inter-group maize hybrids was investigated and reported that sum of marker effects across significantly associated markers provided an estimate for predicting genotypic value of the hybrids in relation with their genetic relatedness Vuylsteke et al., (2000).

\section{Marker based HP in DH lines}

DH technology greatly facilitated and accelerated the development of various crop inbreed lines. Complete homozygosity could be achieved in extremely short time, by haploidization of the genome/chromosome 
and subsequent chromosome doubling. Therefore, DH lines are neither subjected to performance assessment nor to any testing for testcross performance. DH lines represent a random sample of lines from the parental cross because these have not undergone any selection or testcross performance, due to these reasons, the prediction of heterosis with molecular markers is especially attractive for $\mathrm{DH}$ lines as an alternative to expensive field testing and is greatly expected to accelerate the identification of superior hybrids.

\section{Gene expression approach}

Elite rice hybrids and their parents were taken in consideration at three stages of young panicle development, using a cDNA microarray of expressed sequence tags (ESTs), with objective to access patterns of gene expression that was associated with heterosis (Huang et al., 2006). Study revealed that most of the sequences showed hybridization signals in all the genotypes in at least one stage and half of the sequences produced detectable signals in all stages. Correlations of expressed gene/band with hybrid performance, heterosis and heterozygosity formulated little correlation between differential gene expression and hybrid performance. Parent specific expressions appeared to be positively correlated with heterosis and heterozygosity of marker loci. In this connection, it was noted that correlation between general heterozygosity and heterosis revealed in previous studies of the same data set are on the same order of magnitude as the correlations of differential expression with heterosis and heterozygosity.

\section{QTL approach}

Molecular markers is capable of assessing chromosomal positions to individual QTLs, to determine the types and magnitude of gene effects of individual QTLs, and also to determine which parent possesses the positive allele at each QTL. The ability to find an association between a QTL and a molecular marker depends upon the magnitude of the QTL's effect on the trait, the size of the population being studied, and the recombination frequency between the marker and the QTL. Segregating population $\left(\mathrm{F}_{2}\right.$, backcross, recombinant inbred lines, or double haploids) from the crosses between parents differing in expression of particular traits could be used for determination number and location of genes of large effect regulating the particular trait of interest. Once a tight linkage is found between a molecular marker and a gene of interest, the inheritance of the gene can be traced in breeding programs. Molecular markers offered position mapping of QTLs as a powerful tool, linked to genetically architect complex polygenic traits. QTLs have been studied in agronomic, physiological and others traits in plants particularly designed to study the genetic basis of heterosis and inbreeding depression. Fine-mapping of one major QTL on chromosome 5 dissected, this region into two smaller QTL in repulsion phase linkage, which displayed dominant gene action (Zhou et al., 2012).

High correlation observed between heterosis and the heterozygosity, markers confirms the complex genetic basis and role of dominance in heterosis. Co-workers noted QTL showed over-dominance and concluded as combination of QTL exhibited true overdominance or partial to complete dominance, but they are so tightly linked that their individual effects cannot be separate. It is crucial to validate the markers identified from one set of parental lines into a few more sets of parental lines to eliminate genotype effects. Correlations using specific heterozygosity based on small number of positive markers (markers linked to QTLs responsible for 
heterotic yield response) are much more significant than those obtained using markers for general heterozygosity.

\section{Wider compatibility approach}

Successful inter-subspecific crosses in indica and japonica combinations depend on genetic remedy in persisting hybrid semi-sterility problem. Genetic basis of hybrid sterility has been largely investigated. Discovery of wide compatibility system in Ketan Nangka, Dular etc, showed complete panicle existence as well as compatible with both indica and japonica. The sterility neutralizing phenomenon was found to be a simply inherited in the Mendelian fashion. The semi sterility explained through 'single locus allelic interaction' model is referred as HSGL or WCGL. Till date more than 30 hybrid sterility neutralizing gene loci have been identified, like $S 5^{n}, S 8^{n}, S 12^{n}$ etc. and are being used in overcoming the inter-sub-specific hybrid sterility. Conventionally, wide compatibility or otherwise of a variety or breeding line is determined by studying the progeny for spikelet sterility of its crosses with typical indica and japonica testers. Realizing that use of easily identifiable DNA markers linked to the trait would make the process not only identification and characterization of WC gene sources but also for extensive breeding, selection of parental lines easy and reliable; efforts have been to map major WCG-locus specific markers (Sobrizal et al., 2001). Cosegregation analysis of $S-5$ locus indicated RM 276 and RM 225 closely linked to the hybrid sterility, while 65 RM 276 and RM 225 in the vicinity of the locus $S-5$. Highly significant intra-locus allelic interaction has been found in respect of markers located near S-8 (RM 146 and RM 412) and S-6 (RM 136) loci.

The knowledge generated on the various HSG loci has been well utilized worldwide to evolve many parental lines of RPHR with neutral alleles at $S-5$ and $S$-8 are now being used to obtain spikelet fertility in intersubspecific hybrids. Chen et al., (2008) cloned S5, a major locus for indica japonica hybrid sterility and wide compatibility, using a map-based cloning approach and found that S5 encodes an aspartic protease conditioning embryo-sac fertility and a discontinuous 136bp deletion. The same aspartic protease was reported in wide-compatible varieties carrying the neutral allele at S5 locus compared to the indica and japonica alleles using S5 multiplex marker system. Revathi et al., (2010), Sumdaram et al., (2010) and Soni et al., (2016) identified some Indian lines possessing S5 neutral allele could be utilized in inter subspecific hybridization to exploit higher level of heterosis.

\section{High resolution genotyping}

In recent years, various technologies of highresolution genome analysis have been developed which provide an exceptional level of insight into genome structural diversity across the cultivated crop plants. NGS technologies enabled low-cost discovery of sequence variation for all crops and are undergoing further development in terms of costs and efficiency Poland and Rife (2012). These technologies uses diverse methods ranging from genome-scale resequencing or skim sequencing, transcriptome sequencing, reduced representation genotyping-bysequencing, or sequence capture approaches. Reference genome sequences of large and complex genomes have been deciphered by the next-generation sequencing for many important crop species. Sequence data helps to develop high-density, high-throughput genotyping arrays for the assessment of genome wide SNPs, genome wide association studies, genetic mapping, and genomic selection strategies in all major crop species. Even if completed reference sequence of the 
plant species is not available, the reducedrepresentation genotyping-by sequencing (GBS) methods enable use of NGS technologies for high-throughput genomic resequencing, at a constantly shrinking price. Romay et al., (2013) generate almost 7lacs genome wide SNPs in a panel of 2815 diverse inbred lines. Further, assemblies of crop relative genome diversity provide pangenome which gives unprecedented insight into the gene diversity available in secondary crop gene pools Lu et al., (2015). Recently, valuable information on pan-genomic variation to phenotypic variation for important adaptive traits has been identified in soya bean and maize Li et al., (2014).

Exploiting heterosis of hybrid crops for yield gain and stability even in inbreeding crops like bread wheat is the major drivers of increased agricultural production. Highresolution analysis of crop diversity on a chromosomal and sub-genomic scale helps in identifying novel genetic diversity to improve yield and heterosis. High resolution genome profiling of large hybrid populations by the NGS technologies for dissection of loci and genes involved in heterotic expression can shed new light on the genetic background of heterosis. Whole-genome profiling was used in the concept of heterotic haplotype capture (HHC), which identifies and enrich diversitypoor genome regions. Fully sequenced, diverse founder lines are used in HHC to generate large structured pre-breeding populations Gore et al., (2009). HHC enables characterization of novel diversity on a highresolution, sub-chromosomal level, and used to introduce the enriched genomic regions for targeted improvement of heterosis into hybrid breeding programmes Snowdon et al., (2015).

Recently, detailed genome wide sequence analysis of a population of 1495 elite hybrid rice varieties along with their inbred parental lines, was done in order to investigate genomic effects on hybrid vigour for 38 agronomic traits Huang et al., (2015). All the inbred parental lines exhibited $\sim 1.3$ million polymorphic SNP markers which were subsequently used to study population genetic parameters. Heterozygous chromosome regions contributing trait expression in the $\mathrm{F} 1$ hybrids were revealed. The corresponding genomic effects on phenotypic traits demonstrated that that dominance complementation contributes most to the heterosis effect in hybrid rice production. Thus high-throughput, high-density genomeprofiling tools in combination of forward and background selection can be expected to efficiently exploit hybrid vigour which will significantly improve potential for increasing breeding gain. However, the molecular and genetic mechanisms underlying this phenomenon are still not completely understood.

High-throughput genotyping platforms have been established for large plant breeding programs during the past decade. Marker data, generated by routine fingerprinting of new lines, has multiple uses such as assessment of genetic diversity, understanding genetic relatedness and phylogeny of species, raising the ceiling to genetic yield, depending the yield against biotic and abiotic stress and hybrids and their parental lines, mapping and tagging of genes/QTLs of interest for marker assisted selection/backcross breeding, map based cloning of genes and characterization. Multiplex marker platforms analyse thousands of single nucleotide polymorphism (SNP) markers simultaneously in a single step, are available for routine application in breeding of major crops. Recent advances in DH and molecular marker technologies have the potential to further accelerate hybrid breeding programs and improve their efficiency. In addition to molecular markers, transcriptome and metabolomics provided means for heterosis prediction, as was 
demonstrated for transcriptome analysis in maize and metabolome analysis in Arabidopsis. Analysis of the genomic, transcriptomic and metabolomics data in combination with molecular-based prediction of heterosis needs to be exploring for its potential to improve the efficiency of applied hybrid breeding programs.

\section{Future prospects}

Yield is a polygenic trait, and it entails a myriad of physiological and biochemical processes interacting with each other at cellular or whole plant levels. Therefore, a reliable prediction of heterosis will depend on exploitation of multifarious metabolic pathways involving multiple genes or genomic regions (QTLs).

Computer generated simulation data results, suggested that effective prediction of hybrid performance based on marker heterozygosity is possible only if dominance effects are strong, allele frequencies at individual loci in parental inbreeds are negatively correlated, heritability of the traits of interest is high, average parental allele frequencies vary only within a narrow range.

Molecular markers are randomly dispersed or unlinked to QTL. Therefore, it would be better to select, tightly linked QTL-associated markers for the purpose of heterosis prediction.

Advancement in genomics, proteomics and metabolomics has helped to focus research towards the identification of the complete network of genes responsible for different pathways including those of transcription factors.

Such studies in rice, maize and wheat conceptualized development of a heterosisrelated Genes Database (HRGD, http://hrgd.big.ac.cn/index.html), which provides the information of differentially expressed genes and transcription factors associated with heterosis at different growth stages of the crop. This will help in the identification of markers and their employment for a reliable prediction of heterosis. However, the molecular and genetic mechanisms underlying this phenomenon are still not completely understood.

\section{Acknowledgement}

Author would like to thank Dr. Shailesh Tiwari (Research Associate), Division of Genomic Resources, National Bureau of Plant Genetic Resources, Pusa Campus, New Delhi, India, Dr. John T. Newmah (Principal Scientist \& Head) Faculty of Cereal Crop Breeding, Central Agricultural Research Institute, Bong, Liberia. Dr. Innocent DossouAminon, Associate Scientist, Faculty of Science and Technology of Dassa Polytechnic University of Abomey, Dassa-Zoumè, Benin, and Dr.R M Sundaram (Principal Scientist) Indian Institute of Rice Research, Rajendranagar, Hyderabad, Telangana, for valuable suggestions and completion of this manuscript.

\section{Abbreviations}

AFLP: amplified fragment length polymorphism, CMP: Coefficient of polymorphism, EST: expressed sequence tag, GD: genetic distance, GS: Genetic similarity, DH: Double haploid; HP: Heterosis prediction, QTL: quantitative trait loci, RAPD, randomly amplified polymorphic DNA, SSR: simple sequence repeats, MRD: Modified Rogers distance, HSGL: Hybrid Sterility Gene Loci, WCGL: Wide Compatibility Gene Loci, NGS: Next Generation DNA Sequencing, HHC: Heterotic Haplotype Capture, GBS: genotyping-by sequencing. 


\section{References}

Charlesworth, D., and Willis, J.H. 2009 The genetics of inbreeding depression. Nature Reviews Genetics. 10, 783-796.

Chen, H., He, H., Zhou F., Yu, H. and Deng, X.W. 2013 Development of genomicsbased genotyping platforms and their applications in rice breeding. Currt Opn in Pl. Biol. 16, 1-8.

Chen, J., Ding, J., Ouyang, Y., Du, H., Yang, J., Cheng, K. et al., 2008 A triallelic system of S5is a major regulator of the reproductive barrier and compatibility of indica-japonica hybrids in rice. Proc. Natl. Acad. Sci., USA, 105, 1143611441.

Chen, X., Sun D, Rong DF, Sun G and Peng J. 2010 Relationship of genetic diversity and hybrid performance in hybrids derived from a new photoperiod-thermo sensitive male sterile wheat line $337 \mathrm{~S}$. Euphy. 175, 365-371.

Choukan, R. and Warburton, M.L. 2006 Genetic distance based on SSR markers and testcross performance of maize inbred lines. Iranian J. of Biotech. 4, 254-259.

Delucchi, C., Eyhérabide, G.H., Lorea, R.D., Presello, D.A., Otegui, D.E., and López, CG 2012 Classification of argentine maize landraces in heterotic groups. Maydica 57, 26-33.

Gore, M.Q., Chia J.M., Elshire, R.J., Sun, Q., Ersoz, E.S., Hurwitz, B.L. et al., 2009 A first-generation haplotype map of maize. Science. 326, 1115-1117.

Heffner, E.L, Sorrells, M.E., and Jannink, J.L. (2001). Genomic selection for crop improvement. Crop Sci.49: 1-12.

Huang, X., Yang, S., Gong, J., Zhao, Y., Feng, Q., Gong, H. et al., 2015 Genomic analysis of hybrid rice varieties reveals numerous superior alleles that contribute to heterosis. Nat. Comm. 6, 6258.
Huang, Y., Zhang, L., Zhang, J., Yuan, D., $\mathrm{Xu}, \mathrm{C} ., \mathrm{Li}, \mathrm{X}$. et al., 2006 Heterosis and polymorphisms of gene expression in an elite rice Plant hybrid as revealed by a microarray analysis of 9198 unique ESTs. Plt. Mol Biol. 62, 579-591.

Jaikishan, I., Rajendrakumar, P., Ramesha, M.S., Viraktamath, B.C., Balachandran, S.M. and Neeraja, C.N. 2010 Prediction of heterosis for grain yield in rice using 'Key' informative EST-SSR markers. Plant Breed. 129,108-111.

Kashiani, P., Saleh G., Panandam J.M., Abdullah N.A.P. and Selamat A 2012 Molecular characterization of tropical sweet corn inbred lines using microsatellite markers. Maydica. 57, 154-163.

Li, Y.H., Zhou, G. and Ma, J.J. 2014 De novo assembly of soybean wild relatives for pan-genome analysis of diversity and agronomic traits. Nat. Biotech. 32, 1045-1052.

Lu, F., Romay, M.C., Glaubitz, J.C., Bradbury, P.J., Elshire, R.J., Wang, T. et al., 2015 High-resolution genetic mapping of maize pangenome sequence anchors. Nat. Commun. 6, 6914.

Micic, D.I., Drinic, S.M., Nikolic, A., Janic, V.L. 2007 Comparison of AFLP and SSR markers for genetic diversity Studies in maize populations. Maydica. 52, 399-406.

Poland, J.A. and Rife, T.W. 2012 Genotyping-by-Sequencing for plant breeding and genetics. Plant Genome. 5, 92.

Rabbani, M.A., Masood, M.S., Shinwari, Z.K. and Shinozaki, K.Y. 2010 Genetic analysis of basmati and non-basmati Pakistani rice (Oryza sativa L.) Cultivars using Microsatellite markers. Pak. J. Bot. 42, 2551-2564.

Rajendran, A., Muthiah, A., John, J., Shanmugasundaram, P. and Raju, P. 2014 Heterotic grouping and patterning 
of quality protein maize inbreds based on genetic and molecular marker studies. Turk J Biol. 38, 10-20.

Reis, A.J.D., Chaves, L.J., Duarte, J.B. and Brasil, E.M. 2005 Prediction of hybrid means from a partial circulant diallel table using the ordinary least square and the mixed model methods. Genet Mol. Biol. 28, 314-320.

Revathi,, P., Singh, A.K., Sundaram, R.M., Senguttuvel, P., Kemparaju, K.B., Hariprasad et al., 2010 Molecular screening for the presence of wide compatibility gene S5neutral allele in the parental lines of hybrid rice. Indian J. Genet., 70, 373-376.

Romay, M.C., Millard, M.J., Glaubitz, J.C., Peiffer, J.A., Swarts, K.L., Casstevens, T.M. et al., 2013 Comprehensive genotyping of the USA national maize inbred seed bank. Genome Biol. 14, 55.

Selvaraj, I., Nagarajan, P., Thiyagarajan, K. and Bharathi, M. 2010 predicting the relationship between molecular marker heterozygosity and hybrid performance using RAPD markers. Afr. J. of Biotech. 9, 7641-7653.

Shieh and Thseng (2002). Genetic diversity of Tainan-white maize inbred lines and prediction of single cross hybrid performance using RAPD markers. Euphytica124 (3):307-313.

Singh, V.K., Upadhyay, P., Sinha P., Mall, A.K., Kumar, R., Singh, E.A., et al., 2011 Prediction of Hybrid Performance Based on the Genetic Distance of Parental Lines in Two-Line Rice (Oryza sativa L.) Hybrids. J. Crop Sci. Biotech. $14,1-10$.

Snowdon, R.J., Abbadi, A., Kox, T., Schmutzer, T. and Leckband, G. 2015 Heterotic haplotype capture: precision breeding for hybrid performance. Trends in Plant Sci. 20, 410-413.

Sobrizal, Y., Matsuzaki, P. and Yoshimura, A. 2001 Mapping of a gene for pollen semi-sterility on chromosome 8 of rice. Rice Genetics Newsletter 18, 59-61.

Soni, S.K., Yadav, V.K., Bhadana, V.P., Yadav, M.C. and Sundaram, R.M. 2016 Wide compatibility gene approaches and heterosis relationship in japonica $\times$ indica hybrid rice (Oryza sativa L.) Mol. Plt. Breed. 7, 1-12.

Soni, S.K., Yadav, VK, Bhadana, VP, Yadav, M. C., and Sundaram, RM (2017). Prediction of heterosis using hypervariable SSR markers in tropical japonica $x$ indica rice hybrids (Oryza sativa L). International Journal of current microbiology and applied sciences. 6 (10)1419-1427.

Sud, S., Bains, N.S., Nanda, G.S., Singh, K. and Arya, L. 2010 Genetic diversity and heterosis in wheat. SABRAO J. of Breed. and Genet. 42, 95-104.

Sundaram, R.M., Sakthivel, K., Hariprasad, A.S., Ramesha, M.S., Viraktamath, B.C., Neeraja, C.N. et al., 2010 Molecular screening for the presence of wide compatibility gene S5, validation of a PCR-based functional marker system for the major wide-compatible gene locus S5 in rice. Mol. Breed. 26:719-727.

Usatov, A.V., Klimenko, A.I., Azarin, K.V., Gorbachenko, O.F., Markin, N.V., Tikhobaeva, V.E. et al., 2014 The relationship between heterosis and genetic distances based on SSR Markers in helianthus annuus. American J. of Agril and Biol. Sci. 9, 270-276.

Vuylsteke, M., Kuiper, M. and Stam, P. 2000 Chromosomal regions involved in hybrid performance and heterosis: their AFLP-based identification and practical use in prediction model. Heredity 85, 208-218.

Wu, J.W., Hu, C.Y., Shahid, M.Q., Guo, H.B., Zeng, Y.X., Liu, X.D. et al., 2013 Analysis on genetic diversification and heterosis in auto-tetraploid rice. 
Springer. 2, 439

Xangsayasane, P., Xie F., Hernandez, J.E. and Borromeo, T.H. 2010 Hybrid yield heterosis and genetic diversity of IRRI and Lao rice. Field Crop Res. 117, 1823.

Yu, C.Y., Hu, S.W., Zhao, H.X., Guo, A.G. and Sun, G. L. 2005 Genetic distances revealed by morphological characters, isozymes, proteins and RAPD markers and their relationships with hybrid performance in oilseed rape (Brassica napus L.). Theor. Appl. Genet. 110,
511-518.

Zanaty, E.A.F.M., El-Hadary, M.H., Ismail, M. and El-Gammal, A.A. 2013 Genetic diversity of wheat genotypes based on RAPD relative to F1 hybrid Performance. Int. J. Agron. Plt. Prod. 4, 1098-1107.

Zhou, G., Chen, Y., Yao, W., Zhang, C., Xie, W., Hua, J. et al., 2012 Genetic composition of yield heterosis in an elite rice hybrid. PNAS 109, 1584715852.

\section{How to cite this article:}

Sandeep Kumar Soni, Shailesh Tiwari, John T. Newmah, Innocent Dossou-Aminon and Sundaram, R.M. 2018. Prediction of Hybrid Performance in Crop Plants: Molecular and Recent Approaches. Int.J.Curr.Microbiol.App.Sci. 7(01): 98-108. doi: https://doi.org/10.20546/ijcmas.2018.701.012 